\title{
Late physiotherapy rehabilitation changes gait patterns in post-stroke patients
}

\author{
Aline do Socorro Soares Cardoso Almeida ${ }^{1}$, Alex Tadeu Viana da Cruz Júnior ${ }^{1}$, Skarleth Raissa \\ Andrade Candeira ${ }^{1}$, Nathalya Ingrid Cardoso do Nascimento ${ }^{1}$, Ketlin Jaquelline Santana \\ de Castro², Ramon Costa de Lima², Tatiana Generoso Campos Pinho Barroso ${ }^{3}$, \\ Givago da Silva Souza ${ }^{3}$, Bianca Callegari ${ }^{1}$
}

1 Laboratory of Human Motricity, Federal University of Pará, Belém, Pará, Brazil; ${ }^{2}$ Institute of Biological Sciences, Federal University of Pará, Belém, Pará, Brazil; ${ }^{3}$ Tropical Medicine Nucleus, Federal University of Pará, Belém, Pará, Brazil

\section{Summary}

Study aim: To determine whether a physiotherapy protocol improves the electromyographic activation (EA) during the hemiparetic gait in patients with delayed access to rehabilitation.

Material and methods: 40 post-stroke patients underwent clinical evaluation and gait assessment at the time of admission and at the end of treatment.

Results: The anterior leg muscles tibialis anterior and rectus femoris had earlier onset $(\mathrm{p}=0.0001)$.

Conclusion: Electromyographic findings showed altered patterns during the hemiparetic gait cycle, even in patients with delayed access to treatment.

Keywords: Stroke - Hemiparetic - Surface electromyography - Gait assessment - Physiotherapy

\section{Introduction}

Abnormal electrical activity (EA) findings associated with post-stroke gait show alterations in amplitude of muscle activity, and also in timing of muscle activity during the gait cycle $[2,7,11]$. Despite large individual differences among patients, some common findings have been reported. Perry et al. (1995) found absence or reduced EMG amplitude of paretic ankle dorsiflexors during the late swing and early stance phases [12]. They found premature activity of the paretic calf muscles during the terminal swing and initial stance phase. In addition, they found prolonged gastroc-soleus activation that contributed to foot drop associated with inadequate dorsiflexion during swing. They also sometimes found prolonged activation of the posterior and anterior tibialis muscles, contributing to inversion positioning of the ankle during swing $[5,16]$. Although these electromyographic characteristics are known, no reference is found about chronic hemiparetic patients who did not receive rehabilitation after stroke. This report aims to disentangle this conflicting issue, filling the gap and helping to understand what happens to chronic and non-treated patients.

Physical rehabilitation remains the first-line intervention strategy to attenuate chronic impairments of walking, since it promotes brain organization and plasticity after stroke. However, early intervention for post-stroke patients is strongly recommended, and it may modify EMG abnormal patterns in these cases [3, 11]. In developing countries, such as Brazil, the public rehabilitation system is under high demand and has limited infrastructure. This means that patients wait for long periods without treatment, which makes this group different from those commonly studied in the literature. To provide the basis for the development of gait training strategies, it is necessary to understand, in this particular group, which features of muscle control remain stable over the course of stroke recovery, and what aspects are susceptible to modification, even in non-treated and late stages. Since gait recovery is associated with the development of more selective muscle control [7] or new coactivation strategies [11], we believe that the human and financial efforts to provide late physical therapy may be unavailing. In this context, the 
present study aimed to determine whether it is possible to modify EA patterns in chronic patients, years after stroke. Our hypothesis is that, due to the long time after stroke and delayed access to treatment, gait impairment is set in a way that is no longer responsive to the conventional intervention.

\section{Material and methods}

Forty stroke patients with an average age of 57.43 $( \pm 10.24)$ years ( 14 women and $26 \mathrm{men}$ ) participated in the study. The average weight of the participants was $68.90 \mathrm{~kg}$ $( \pm 10.53 \mathrm{~kg})$ and average height $159.85 \mathrm{~cm}( \pm 8.29 \mathrm{~cm})$. Stroke participants were recruited based on the following criteria: The participants had experienced a unilateral stroke that was hemorrhagic or ischemic and that involved the motor cortex or sub-cortical areas. They were enrolled if they had lower extremity Brunnstrom stage [1] between 3 and 4, and were able to undertake independent walking, defined as the ability to walk ten meters without any assistance (supervision, orthoses or assistive devices). They were not under any physical therapy or following an exercise program prior to enrolment in the study. Participants were excluded if they were unable to follow verbal requests, had other neurological, musculoskeletal or orthopedic conditions, or if they were on antispastic medication at the time of the study. This study has been approved by the internal ethics committee of the Federal University of Pará (report \#141.605).

\section{Procedures}

All patients underwent a clinical examination by an experienced physiotherapist, prior to any experimental procedure. The baseline characteristics assessed were age, sex, time since last stroke onset episode, stroke type, and affected body side.

The patients were evaluated at the time of admission to the physiotherapy (pre-physiotherapy stage) and after the treatment (post-physiotherapy stage). The physiotherapy intervention consisted of 20 sessions of 60 minutes carried out following standard protocols without any intervention from the researchers. This number of sessions is the standard of the public health system due to the great amount of patients. It was performed three times per week, and although the treatment was similar for all subjects, they participated individually in their sessions. Exercises were performed in series of ten repetitions, or 5 minutes (with a 1-minute rest period), and all subjects complied with the treatment protocol, with stretching, passive, active assistive and active range of motion, resistance training, functional electrical stimulation, coordination and balance activities and conventional treadmill training.

\section{Gait evaluation}

Subjects were instructed to walk along a seven-meter flat walkway, made of ethylene vinyl acetate (EVA). Volunteers were asked to walk barefoot using their own natural self-selected speed. We assessed the gastrocnemius lateralis (GAS), tibialis anterior (TA), semitendinosus (ST), biceps femoris $(\mathrm{BF})$, and rectus femoris (RF) muscles on the affected limb. Data from 18 steps were analyzed for the calculations.

\section{Equipment}

Muscle activity was recorded using an eight-channel electromyograph (EMG System do Brasil, São José dos Campos, Brazil). The electromyograph had an amplification of 20 times, band-pass filtered $(20-500 \mathrm{~Hz})$, analogdigital converter with 16 bits of resolution, a sampling rate of $2 \mathrm{kHz}$, and an input range from -12 to $+12 \mathrm{~V}$. Each channel was coupled to two active bipolar circular Ag/ $\mathrm{AgCl}$ electrodes (Meditrace, Mansfield, USA). All procedures were conducted according to the recommendations of the SENIAM project regarding skin preparation, position of the patient and testing connection [4].

The EMG data were analyzed using MATLAB software (Matlab 2008a, MathWorks Inc., MA, USA). The EA were quantified using root mean square (RMS) over a $300 \mathrm{~ms}$ window. The gait data were normalized by the maximum voluntary isometric contraction (MVC) for each muscle. Three tests of MVC were performed, and the average values were used for normalization of the muscles' electrical activity detected during the walking test [6]. After this, the linear envelope of each muscle was calculated using a second order Butterworth low-pass filter (6 Hz cut-off).

Time normalization of EMG gait data to a percentage of the gait cycle was performed assuming that gait cycles are periodic. The times were assigned relative to consecutive ipsilateral hindfoot strikes ranging from $0 \%$ and $100 \%$ of the gait cycle. This was performed using a foot switch (Heel/Toe strike transducer, Emgsystem, São Jose dos Campos, Brazil) that was synchronized to the acquisition system to identify the exact time of initial contact and loss of foot contact with the ground, thereby determining cycle phases. In order to detect muscular activation, we used a criterion for establishing onset and offset activation based on the moment when the EMG signal exceeded $30 \%$ of the minimum amplitude of the signal average per individual [6].

\section{Statistical analysis}

The data were tested for normality using the ShapiroWilk test. Normally distributed variables were compared using Student's t-test. Variables not normally distributed were compared using the Mann-Whitney U test. The significance level was set at 5\%. All tests were conducted using R studio (RStudio, Boston, MA, USA). 


\section{Results}

Physiotherapy was delivered for our patients 5.08 years ( \pm 3.51 years) after the stroke event, which means a delay from what the literature recommends. The average number of subsequent stroke episodes was 1.55 ( \pm 0.78$)$. As commonly described in the epidemiology of the disease, the majority of patients $(50 \%)$ were affected by ischemic stroke, $27.5 \%$ by hemorrhagic stroke, and $22.5 \%$ by both. The right hemisphere of the brain was affected in $62.5 \%$ $(\mathrm{n}=25)$ of the patients and the remaining $37.5 \%(\mathrm{n}=15)$ were affected in the left hemisphere.

\section{Gait assessment}

Physiotherapy leads to a different pattern of muscular recruitment during the swing phase for the anterior muscles (RF and TA), which started their recruitment (onset) earlier (Fig. 1). BF-RF and TA-GAS muscle co-activation was seen during the whole gait cycle, and especially during the stance phase it is longer than the normal patterns described in the literature.

In addition, a spatiotemporal analysis of the gait cycle found no differences in the gait velocity or in the duration of the phases between the pre physiotherapy stage (stance: $56 \pm 12.2 \%$ and $58.4 \pm 10.3$; swing: $42.5 \pm 11.7 \%$ and $43 \pm 11.0 \%$, pre- and post-physiotherapy respectively).

\section{Discussion}

We found that chronic patients had EA altered after 20 sessions, and although they continued to exhibit abnormalities in the temporal characteristics of muscle activity compared to normal gait, these changes in EA deserve attention as subclinical responses to the treatment.

We observed that the TA and RF muscles modified their temporal patterning after physiotherapy intervention and had earlier activation in the swing phase. This may reflect a new strategy adopted by the anterior leg muscles in an attempt to modify the inadequate dorsiflexion and inversion positioning of the ankle during the swing phase in stroke patients $[10,11,12]$. In addition, the duration of BF-RF and TA-GAS muscle co-activation was longer during the stance phase as compared to published data for normal gait $[5,16]$. During the stance phase, the BF muscle is working to help hip extension, whereas the RF muscles' main function should be knee extension [11]. When both muscles are co-activated during the stance phase, this increases the stiffness of the knee and hip joint to help support the full body weight. This compensation may occur due to weakness of the calf muscles [11]. While it does not exist in the normal gait, in our delayed treatment stroke patients, TA-GAS coactivation was important at the beginning of the swing phase to maintain dorsiflexion for the initial contact, and to control foot drop $[11,12]$. This prolonged activation of the posterior and anterior lower leg muscles contributes to inversion of the ankle in our stroke patients, as has been previously reported $[2,3,16]$. It was observed at the post-physiotherapy stage that BF-RF and TA-GAS muscle co-activation was unchanged.

Den Otter [2] compared clinical, spatiotemporal, and electromyographic assessments of post-stroke patients at four time stages during recovery. Although the investigation was performed during early rehabilitation (35 to 90 days after stroke), the results regarding the timing behavior of muscles agreed with ours. They found longer

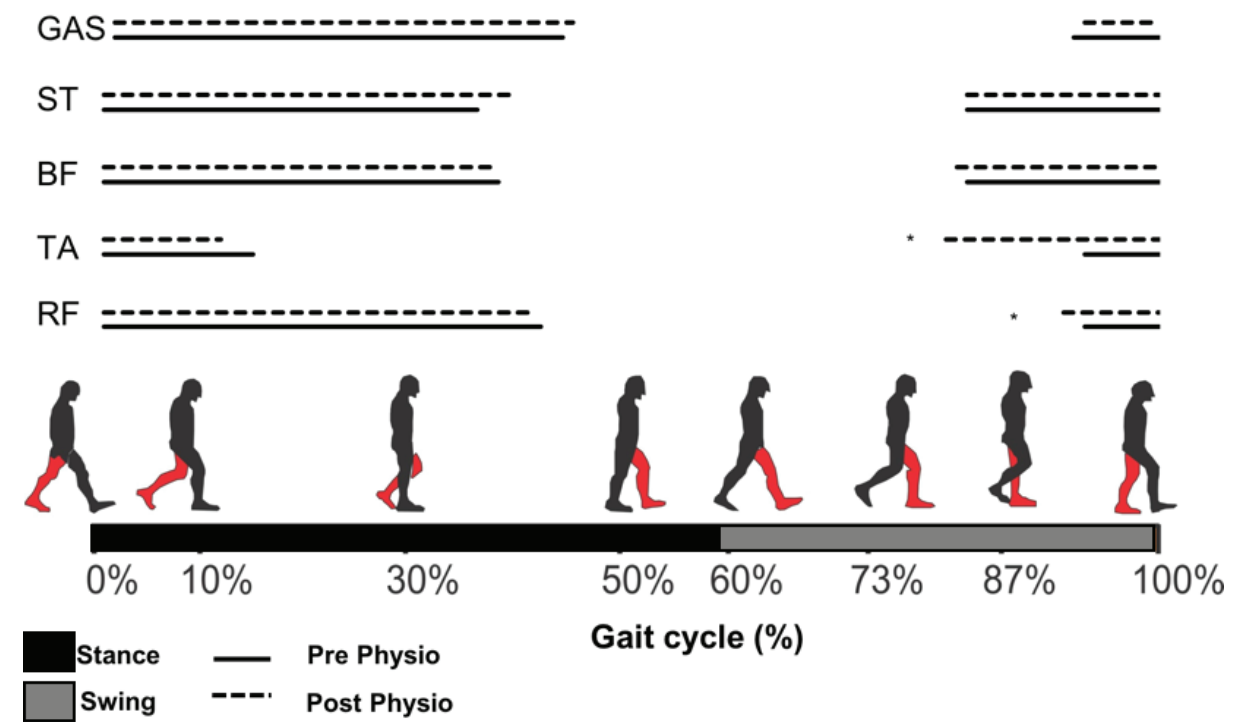

Fig. 1. Muscle timing activation during the gait cycle in pre and post physiotherapy stages. Continuous lines represent muscle activation at the pre-physiotherapy stage. Dashed lines represent muscle activation at the post-physiotherapy stage 
durations of activity in the $\mathrm{BF}$ muscle during the single support phase. They also found a longer activity time of the GAS muscle during the first double support phase, and prolongation of the activity in the RF muscle during the stance phase. They found longer duration of BF-RF muscle co-activation in the paretic group than in controls. After almost 60 days of physiotherapy intervention, the patients' spatiotemporal and clinical measures of mobility and ambulatory independence scores increased significantly but aberrant muscle timing patterns persisted. They suggested that normal temporal patterning of lower extremity muscle activity is not a prerequisite for functional gait recovery $[2,3]$. We found that our patients exhibited some changes in muscle pattern activation after the intervention. Yet, how functional performance is linked to neuromuscular timing patterns, and how these patterns remain stable, or not, over the course of recovery, remain unclear. Apparently, other factors not linked to muscle temporal organization, such as force and spasticity, may be better improved using physiotherapy treatment $[2,3]$.

There is a consensus that the rehabilitation program should be started as soon as possible for better results in post-stroke patients $[9,15,18,19]$. However, no optimal time window has been defined for specific post-stroke rehabilitation interventions [18]. Some studies have suggested that stroke rehabilitation is most effective and improves functional outcomes if initiated early (within three to 30 days after stroke) $[13,14,17]$. On the other hand, there are authors [8] who reported that even chronic stroke patients had functional gains. Whitall et al. (2000) [20] studied bilateral arm training in 14 patients with chronic hemiparetic stroke with a median time after stroke of 30 months. They found that their patients had improved functional motor performance and the benefits were sustained eight weeks after training cessation. This previously described "late plasticity" may explain the modified EMG patterns in our chronic patients although our initial hypothesis was that the gait impairment would be totally non-responsive to intervention.

EMG measurements are effective to understand muscular recruitment patterns and help with the limitation of the standardized clinical scales. However, one of the limitations of our EMG technique was the low number of channels, which allowed five muscles to be achieved and only on the paretic limb. Finally, a control group would be necessary to establish any real gains and help to determine a mechanism for improving locomotor control.

\section{Conclusion}

After undergoing delayed physiotherapy treatment, stroke patients had improved RF and TA muscle onset anticipation, during the swing phase.
Conflict of interest: Authors state no conflict of interest.

\section{References}

1. Brunnstrom S. (1966) Motor testing procedures in hemiplegia: based on sequential recovery stages. Phys. Ther, USA, 46(4): 357-375.

2. Den Otter A.R., Geurts A.C.H., Mulder T., Duysens J. (2006) Gait recovery is not associated with changes in the temporal patterning of muscle activity during treadmill walking in patients with post-stroke hemiparesis. Clin. Neurophysiol., 117(1): 4-15. DOI: 10.1016/j. clinph.2005.08.014.

3. Den Otter A.R., Geurts A.C.H., Mulder T., Duysens J. (2007) Abnormalities in the temporal patterning of lower extremity muscle activity in hemiparetic gait. Gait Posture, 25(3): 342-352. DOI: 10.1016/j.gaitpost.2006.04.007.

4. Hermens H.J., Freriks B., Disselhorst-Klug C., Rau G. (2000) Development of recommendations for SEMG sensors and sensor placement procedures. J. Electromyogr. Kines., 10(5): 361-374. DOI: 10.1016/S1050-6411(00)00027-4.

5. Hirschberg G.G., Nathanson M. (1952) Electromyographic recording of muscular activity in normal and spastic gaits. Arch. Phys. Med. Rehabil., 33(4): 217-25. Available at: http://www.ncbi.nlm.nih.gov/pubmed/14924804 (Accessed: 11 May 2016).

6. Ingrid Cardoso do Nascimento N., Sepêda Saraiva T., Tadeu Viana da Cruz Jr A., da Silva Souza G. Callegari B. (2014) Barefoot and High-Heeled Gait: Changes in Muscles Activation Patterns. Health, 6(6): 2190-2196. DOI: 10.4236/health.2014.616254.

7. Lamontagne A., Richards C.L., Malouin F. (2000) Coactivation during gait as an adaptive behavior after stroke. J. Electromyogr. Kines., 10(6): 407-415. Available at: http://www.ncbi.nlm.nih.gov/pubmed/11102843 (Accessed: 11 May 2016).

8. Liepert J., Miltner W.H.R., Bauder H., Sommer M., Dettmers C., Taub E., Weiller C. (1998) Motor cortex plasticity during constraint,induced movement therapy in stroke patients. Neurosci. Lett., 250(1): 5-8. DOI: 10.1016/ S0304-3940(98)00386-3.

9. Maulden S.A., Gassaway J., Horn S.D., Smout R.J., DeJong G. (2005) Timing of initiation of rehabilitation after stroke. Arch. Phys. Med. Rehabil., 86(12 SUPPL. 2): S34-S40. DOI: 10.1016/j.apmr.2005.08.119.

10. Moore S., Schurr K., Wales A., Moseley A., Herbert R. (1993) Observation and analysis of hemiplegic gait: swing phase, Aust. J. Physiother., 39(4): 271-278. DOI: 10.1016/S0004-9514(14)60487-6.

11. Peat M., Dubo H.I., Winter D.A., Quanbury A.O., Steinke T., Grahame R. (1976) Electromyographic temporal analysis of gait: hemiplegic locomotion. Arch. Phys. Med. 
Rehabil., 57(9): 421-5. Available at: http://www.ncbi. nlm.nih.gov/pubmed/962569 (Accessed: 11 May 2016).

12. Perry J. (1993) Determinants of muscle function in the spastic lower extremity. Clin. Orthop. Relat. Res., 288: 10-26. Available at: http://www.ncbi.nlm.nih.gov/ pubmed/8458121 (Accessed: 11 May 2016).

13. Piassaroli P.A., Almeida G.C., Luvizotto J.C., Suzan A.B. (2012) Physical Therapy Rehabilitation Models in Adult Patients with Ischemic Stroke Sequel, Revista Neurociências, 20(1): 128-137.

14. Pollock A., Baer G., Campbell P., Choo P.L., Forster A., Morris J., Pomeroy V.M. Langhorne P. (2014) Physical rehabilitation approaches for the recovery of function and mobility following stroke. [In:] Pollock A. (ed.) Cochrane Database of Systematic Reviews. Chichester, UK: John Wiley \& Sons, Ltd. DOI: 10.1002/14651858. CD001920.pub3.

15. Routson R.L., Clark D.J., Bowden M.G., Kautz S.A., Neptune R.R. (2013) The influence of locomotor rehabilitation on module quality and post-stroke hemiparetic walking performance, Gait Posture, 38(3): 511-517. DOI: 10.1016/j.gaitpost.2013.01.020.

16. Shiavi R., Bugle H.J., Limbird T. (1987) Electromyographic gait assessment, part 2: Preliminary assessment of hemiparetic synergy patterns*, J. Rehabil. Res. Dev., 24(2): 24-30.

17. Suh J.H., Han S.J., Jeon S.Y., Kim H.J., Lee J.E., Yoon T.S., Chong H.J. (2014) Effect of rhythmic auditory stimulation on gait and balance in hemiplegic stroke patients. Neurorehabil., 34(1): 193-199. DOI: 10.3233/NRE131008 .
18. Teasell R., Bitensky J., Salter K., Bayona N. (2005) The role of timing and intensity of rehabilitation therapies. Top. Stroke Rehabil., 12(3): 46-57. DOI: 10.1310/ETDP6DR4-D617-VMVF.

19. Urban K., Ignasiak Z., Wronecki K., Skrzek A. (2015) Change in movement patterns asymmetry in infants with central coordination disorder in continuous studies, Biomed. Hum. Kinet., 7: 156-162. DOI: 10.1515/bhk-20150023 .

20. Whitall J., Waller S.M., Silver K.H.C., Macko R.F., McCombe Waller S., Silver K.H.C., Macko R.F., Waller S.M., Silver K.H.C., Macko R.F. (2000) Repetitive Bilateral Arm Training With Rhythmic Auditory Cueing Improves Motor Function in Chronic Hemiparetic Stroke. Stroke, 31(10): 2390-2395. DOI: 10.1161/01.STR.31.10.2390.

Received 21.11.2016
Accepted 21.02.2017

(C) University of Physical Education, Warsaw, Poland

\section{Acknowledgments}

This research was supported by the following grants: Pará Amazon Research Support Foundation (FAPESPA) number180/2012; CAPES/COFECUBE research grant number 819-14 and research rectory from UFPA. ASSCA, ATVCJ, SRAC and NICN were recipient of a graduate student Fellowship. The funders had no role in study design, data collection and analysis, decision to publish, or preparation of the manuscript. 